\title{
GOCE: assessment of GPS-only gravity field determination
}

\author{
Adrian Jäggi · H. Bock · U. Meyer • \\ G. Beutler · J. van den IJssel
}

Received: 18 February 2014 / Accepted: 27 August 2014 / Published online: 10 September 2014

(C) Springer-Verlag Berlin Heidelberg 2014

\begin{abstract}
The GOCE satellite was orbiting the Earth in a Sun-synchronous orbit at a very low altitude for more than 4 years. This low orbit and the availability of high-quality data make it worthwhile to assess the contribution of GOCE GPS data to the recovery of both the static and time-variable gravity fields. We use the kinematic positions of the official GOCE precise science orbit (PSO) product to perform gravity field determination using the Celestial Mechanics Approach. The generated gravity field solutions reveal severe systematic errors centered along the geomagnetic equator. Their size is significantly coupled with the ionospheric density and thus generally increasing over the mission period. The systematic errors may be traced back to the kinematic positions of the PSO product and eventually to the ionosphere-free GPS carrier phase observations used for orbit determination. As they cannot be explained by the current higher order ionospheric correction model recommended by the IERS Conventions 2010, an empirical approach is presented by discarding GPS data affected by large ionospheric changes. Such a measure yields a strong reduction of the systematic errors along the geomagnetic equator in the gravity field recovery, and only marginally reduces the set of useable kinematic positions by at maximum $6 \%$ for severe ionosphere conditions. Eventually it is shown that GOCE gravity field solutions based on kinematic positions have a limited sensitivity to the largest annual signal related to land hydrology.
\end{abstract}

\footnotetext{
A. Jäggi $(\varangle) \cdot$ H. Bock $\cdot$ U. Meyer $\cdot$ G. Beutler Astronomical Institute of the University of Bern, Sidlerstrasse 5, 3012 Bern, Switzerland

e-mail: adrian.jaeggi@aiub.unibe.ch

J. van den IJssel

Faculty of Aerospace Engineering, Delft University of Technology, Kluyverweg 1, 2629 HS Delft, The Netherlands
}

Keywords GOCE · GPS · Gravity field · Accelerometer data - Geomagnetic equator - Higher-order ionosphere (HOI) - Time variable gravity

\section{Introduction}

Precise orbit determination (POD) of low Earth orbiters (LEOs) using global positioning system (GPS) data is required to solve for the long-wavelength part of the Earth's gravity field. This is evident for missions uniquely relying on the GPS high-low Satellite-to-Satellite Tracking (hl-SST) technique such as the past gravity mission CHAllenging Minisatellite Payload (CHAMP, cf. Reigber et al. 1998) or the recently launched magnetic field mission Swarm (ESA 2004). GPS-based POD is also important for the Gravity field and steady-state Ocean Circulation Explorer (GOCE, cf. Drinkwater et al. 2006) mission, where the low-degree coefficients of GOCE-only gravity field solutions are exclusively determined from GPS data, because the measurements of the core instrument, the three axis gravity gradiometer, are band-limited (Pail et al. 2011). For gravity missions such as the Gravity Recovery And Climate Experiment (GRACE, cf. Tapley et al. 2004), where the recovery of most coefficients is dominated by the K-Band low-low Satellite-to-Satellite Tracking (ll-SST) technique (e.g., Beutler et al. 2010b), GPS-based POD plays a less pronounced role.

The GOCE satellite was orbiting the Earth in a Sunsynchronous orbit at a very low orbital altitude for more than 4 years. Since the start of the nominal mission phase on November 1, 2009 until July 31, 2012 the orbital altitude was maintained by a drag-free flight at $254.9 \mathrm{~km}$, which corresponds to a repeat cycle of 979 revolutions in 61 days (Floberghagen et al. 2011). The satellite was 
equipped with two high-quality 12-channel dual-frequency Lagrange receivers with helix-antennas for GPS hl-SST (Intelisano et al. 2008), and six high-quality accelerometers (Cesare 2008) forming the GOCE core-instrument, the three-axis gravity gradiometer (Rummel et al. 2011). The low orbital altitude of the satellite and the availability of more than 4 years of high-quality instrumental data make the GOCE mission an interesting test case to assess the contribution of GPS hl-SST to the recovery of both the static and time-variable gravity field. The lessons learned from this study may be beneficial for the exploitation of GPS hl-SST for gravity field determination from non-dedicated gravity missions such as the recently launched Swarm mission. In view of the possibility of a potential failure of the GRACE spacecraft in the near future, currently being the only dedicated gravity mission in orbit, GPS hl-SST might play an important role to bridge the gap between the current GRACE and the future GRACE Follow-On mission (Flechtner et al. 2013).

GOCE $1 \mathrm{~s}$ kinematic positions are determined at the observation epochs of the GPS data of the main receiver/antenna unit in the framework of the GOCE High-level Processing Facility (HPF, Koop et al. 2006) as part of the GOCE precise science orbit product (PSO, Bock et al. 2011a) by a precise point positioning approach (Švehla and Rothacher 2005). Kinematic LEO positions are independent of the LEO dynamics and therefore well suited as pseudo-observations for an independent recovery of the long-wavelength part of the Earth's gravity field (e.g., Jäggi et al. 2008). GOCE 1 s kinematic positions of the PSO product are used by various groups adopting different approaches for long-wavelength gravity field determination (e.g., Baur et al. 2014). The quality of the recovery critically depends on the quality of the kinematic positions. In order to fully exploit long-wavelength gravity field recovery from kinematic GOCE positions, a precise modeling of systematic GOCE GPS carrier phase errors, e.g., antenna phase center variations of the receiving antenna, is mandatory in the precise point positioning process (e.g., Jäggi et al. 2009; Bock et al. 2011b). A clearly variable quality of the kinematic positions over the entire GOCE mission duration was found despite all these measures by Bock et al. (2014). The impact on the quality of GOCE GPS-only gravity field recovery is assessed in this article, where special emphasis is put on the further investigation (and removal) of systematic orbit errors and the assessment of their propagation to GOCE GPS-only gravity field solutions. Eventually, the impact of unmodeled time-variable gravity signals on GOCE GPS-only gravity field recovery is assessed.

Section 2 briefly introduces the adopted methods for gravity field determination based on kinematic positions. Section 3 presents gravity field results based on the official GOCE PSO products and analyzes observed shortcomings. Systematic errors along the geomagnetic equator are further analyzed in Sect. 4, where gravity field recoveries based on new orbits are presented, as well. The potential of recovering time variable signals from GOCE kinematic positions is eventually assessed in Sect. 5.

\section{Methods for GPS-only gravity field recovery}

Several approaches may be used to perform gravity field determination from GOCE kinematic positions (Baur et al. 2014). In this article the Celestial Mechanics Approach is used to solve a generalized orbit determination problem as already discussed in detail by Jäggi et al. (2011a). In a first step a priori orbits are computed based on the kinematic positions serving as pseudo-observations. The latter are weighted according to their covariance information obtained from the kinematic point positioning (Jäggi et al. 2011b) and are fitted over $24 \mathrm{~h}$ arcs by numerically integrating the equation of motion defined by the a priori force models (see Table 1), and by adjusting arc-specific orbit parameters. In order to demonstrate the strength of our approach the gravity field model EGM96 (Lemoine et al. 1997) from the pre-CHAMP era was selected as a priori gravity field model and the GOCE

Table 1 Data, models, and parameters used for the standard GPS-only gravity field determination

\begin{tabular}{|c|c|}
\hline Kinematic positions & $\begin{array}{l}\text { GOCE SST_PKI product, }^{\mathrm{a}} \\
24 \mathrm{~h}, 1 \mathrm{~s} \text { sampling }\end{array}$ \\
\hline Position weights & $\begin{array}{l}\text { GOCE SST_PCV product, }{ }^{\mathrm{a}} \\
\text { covariances over } 4 \mathrm{~s}\end{array}$ \\
\hline Accelerometer data & $\begin{array}{l}\text { GOCE EGG_CCD product } \\
\text { (used for selected } \\
\text { solutions) }\end{array}$ \\
\hline Gravity field model & EGM96 $^{\mathrm{c}}(120 \times 120)$ \\
\hline Solid Earth and pole tides & ${\text { IERS } 2003^{\mathrm{d}}}$ \\
\hline Ocean tides & FES2004 $(30 \times 30)$ \\
\hline Luni-solar-planetary gravity & $\mathrm{DE} 405^{\mathrm{f}}$ \\
\hline $\begin{array}{l}\text { Atmosphere and } \\
\text { Ocean Dealiasing }\end{array}$ & $\begin{array}{l}\text { AOD1B RL05量 (used for } \\
\text { selected solutions) }\end{array}$ \\
\hline \multirow[t]{2}{*}{ Empirical parameters } & $\begin{array}{l}\text { Constant accelerations per } \\
24 \mathrm{~h} \text { arc in R,A,O }\end{array}$ \\
\hline & $\begin{array}{l}\text { Pseudo-stochastic pulses (constrained) } \\
\text { in R,A,O every } 6 \text { min }\end{array}$ \\
\hline Reference frame & IERS2003 \\
\hline transformation & CODE final ERPs ${ }^{\mathrm{h}}$ \\
\hline $\begin{array}{l}\text { a EGG-C (2010b) } \\
\text { b ESA (2006) } \\
\text { c Lemoine et al. (1997) } \\
\text { d McCarthy and Petit (2004) } \\
\text { e Lyard et al. (2006) } \\
{ }^{\text {d }} \text { Standish (1998) } \\
\text { g Flechtner et al. (2006) } \\
\text { h Dach et al. (2009) }\end{array}$ & \\
\hline
\end{tabular}


accelerometer data were not taken into account. The impact of the GOCE accelerometer data for GPS-based gravity field determination is separately addressed later on in this article in Sect. 3.1. Apart from the six Keplerian elements, constant and once-per-revolution empirical accelerations acting over the entire daily arcs are set up in the radial (R), alongtrack (A), and out-of-plane (O) directions to compensate for the main part of the unmodeled non-gravitational perturbations. Remaining deficiencies are absorbed by estimating additional pseudo-stochastic pulses (instantaneous velocity changes) or accelerations (piecewise constant) at predefined epochs for the R, A, O directions. As pseudo-stochastic pulses do not affect the satellite trajectory in-between the pulse epochs (Jäggi et al. 2006) they are thought to be slightly better suited for gravity field recovery and therefore serve as the type of pseudo-stochastic parameters used in this study. A priori assumed variances (constraints) may be imposed on these parameters on request.

Based on the computed a priori orbits gravity field determination from kinematic positions is set up as a generalized orbit improvement problem (Beutler et al. 2010a). The actual orbits are expressed as truncated Taylor series with respect to all unknown parameters, i.e., with respect to the arc-specific orbit parameters and the spherical harmonic ( $\mathrm{SH})$ coefficients, about the a priori orbits. Based on the partial derivatives with respect to all parameters daily normal equations (NEQs) are set up for all parameters according to standard least-squares adjustment. Arc-specific parameters are then pre-eliminated before the daily NEQs are accumulated into NEQs covering longer time spans. The accumulated NEQ matrix is eventually inverted in order to obtain the corrections of the SH coefficients with respect to the a priori gravity field coefficients and the associated full covariance information. No regularizations are applied to compute the gravity field solutions presented in this article.

\subsection{Parameter constraining and use of accelerometer data}

Bock et al. (2014) showed that realistic constraints imposed on the pseudo-stochastic parameters have a positive impact on the quality of the resulting reduced-dynamic orbits, provided that state-of-the-art background models are used. As the pseudo-stochastic orbit parameters are, together with the gravity field parameters and other empirical parameters, part of the force model, one may not only expect a positive impact on the orbits but also on the derived gravity fields. Several options of constraining are subsequently discussed. They are all applied on the NEQ level and not on the level of observation equations. As a consequence, their implementation is very efficient and ensembles of solutions may be generated.

\subsubsection{Constraining pseudo-stochastic parameters to zero}

In order to simplify the discussion we assume for the moment that pseudo-stochastic parameters are set up as piecewise constant accelerations instead of pulses. As accelerometer data are not used for the generation of the a priori orbits, one may assume that this neglect represents the most pronounced shortcoming of the force model (because the gravity field model is improved in the parameter estimation process). Realistic a priori variances in the $\mathrm{R}, \mathrm{A}, \mathrm{O}$ directions may therefore be best derived from the high-quality GOCE common-mode accelerometer data. By splitting up each arc into subintervals of equal length as the subintervals of the piecewise constant accelerations, mean accelerations may be calculated separately from the accelerometer data [centered around zero, scale factors assumed to be one (Bouman et al. 2011)] for each subinterval and for each component. Eventually the variances of these mean accelerations (binned values) may be computed for each arc and may be used for an arc-specific constraining of the piecewise constant accelerations. Note that constraints for the differences of subsequent accelerations may be derived in analogy by analyzing the differences of the mean accelerations of subsequent intervals. If pulses are used as pseudo-stochastic parameters, the roots of the a priori variances derived from the accelerometer data are simply multiplied by the spacing $\Delta t$ of the subintervals, because approximately a pulse is $p_{i}=\Delta t \cdot \alpha_{i}$, where $\alpha_{i}$ is the corresponding acceleration (Beutler et al. 2010a).

\subsubsection{Constraining pseudo-stochastic parameters to measured accelerations}

A further sophistication of the orbit model is achieved by not just imposing constraints on the pseudo-stochastic parameters derived from accelerometer data, but by taking into account the accelerometer data as part of the force model and by adapting the constraints of the pseudo-stochastic parameters accordingly. Usually this is done right at the beginning when numerically integrating the equation of motion for the generation of the a priori orbits (e.g., Helleputte and Visser 2008; Bock et al. 2014). It is, however, also possible to introduce accelerometer data on the level of already existing NEQs by introducing the binned values from the accelerometer data as additional pseudo-observations with user-specified a priori variances imposed to the piecewise constant accelerations.

Let us assume that $a_{i}$ is the bin value associated with a particular component of the accelerations and with bin number $i$ of the accelerometer data and furthermore that $\alpha_{i}$ is the corresponding estimated value of the piecewise constant acceleration, the following pseudo-observation equations

$1 \cdot \alpha_{i}-a_{i}=v_{i}, \quad i=1, \ldots, n_{a}$ 
may be defined, where $n_{a}$ is the number of piecewise constant accelerations and $v_{i}$ denotes the residual associated with bin number $i$. In matrix form these pseudo-observation equations may be written as

$\mathbf{I} \alpha-\mathbf{a}=\mathbf{v}$,

where $\mathbf{I}$ is the identity matrix, reflecting a particularly simple coefficient matrix to form the linear combination of the estimated parameters $\alpha$.

Let us furthermore assume that $\mathbf{P}$ denotes the diagonal weight matrix associated with the pseudo-observations (2), the corresponding NEQ contribution eventually reads as

$$
\begin{aligned}
\mathbf{I}^{T} \mathbf{P} \mathbf{I} \alpha & =\mathbf{I}^{T} \mathbf{P} \mathbf{a} \\
\mathbf{P} \alpha & =\mathbf{P} \mathbf{a} .
\end{aligned}
$$

This NEQ system is diagonal (absolute constraining) and has to be superimposed to the already existing NEQ system derived from the kinematic pseudo-observations, which already contains the parameter array $\alpha$. Equation (3) is thus merely a generalization of imposing weights on parameters, where not only the normal equation matrix but also the right-hand side of the NEQ system needs to be modified. More complicated regularization schemes, e.g., relative constraining where differences of subsequent parameters are constrained, can be realized accordingly by modifying Eq. (2) and the associated weight matrix $\mathbf{P}$.

The use of the binned acceleration values as pseudoobservations together with already existing NEQs generated without taking accelerometer data into account is particularly attractive for gravity field determination, because the time-consuming generation of NEQs has not to be repeated. Instead the "old" NEQs, containing no (or removable) constraints, just need to be superimposed with the NEQ constituent (3). The resulting orbits and the gravity field must be almost equivalent to analyzing NEQs with accelerometer data used right at the beginning and adding constraints only to the NEQ matrix. The differences consist of neglecting the variations of the accelerometer data within the bins and small non-linear terms.

\section{GOCE GPS-only gravity field recovery using the kinematic GOCE PSO product}

Starting on November 1, 2009, the $1 \mathrm{~s}$ kinematic positions of the GOCE PSO product are used to compute GOCE GPSonly gravity field solutions up to degree and order (d/o) 120 without applying any regularization and without the use of accelerometer data. A long-term solution based on data until July 31, 2012 (period of the Release 4 GOCE models) was generated, as well as solutions based on bi-monthly periods covering November-December of the years 2009, 2010, 2011 , and 2012, respectively. The long-term solution is based

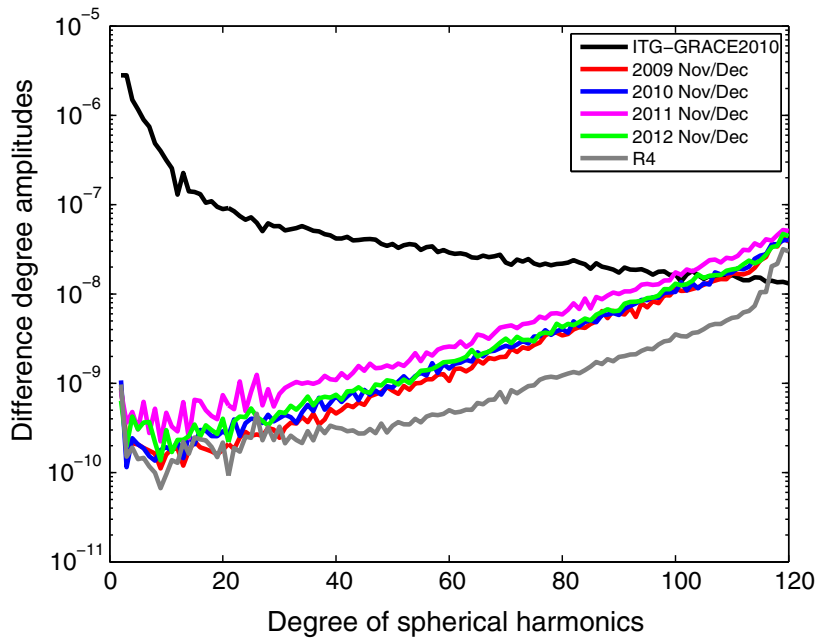

Fig. 1 Square-roots of degree difference variances of gravity field recoveries from GOCE kinematic positions covering different time periods (zonal and near-zonal coefficients excluded) with respect to ITGGRACE2010

on the analysis of a total of 921 (out of 1004 possible) daily arcs, where most of the missing data are related to the socalled GOCE anomaly in 2010 when no data were available (Floberghagen et al. 2011). The bi-monthly solutions are almost complete with only a few days missing.

Figure 1 shows the square-roots of the degree difference variances of the GPS-only solutions up to d/o 120 with respect to the superior gravity field model ITG-GRACE2010 (mainly based on ultra-precise inter-satellite K-band ranging data, Mayer-Gürr et al. 2010). Due to the polar gap caused by the Sun-synchronous GOCE orbit, zonal and near-zonal coefficients are excluded in the comparison according to the rule of thumb by Gelderen and Koop (1997). Based on the significantly different level of the bi-monthly solutions in Fig. 1, it becomes evident that the quality of the individual contributions to the long-term solution is strongly varying. A variable quality of the GOCE kinematic positions was also noted in Fig. 6 from Bock et al. (2014), which is caused by a higher and variable noise over the geomagnetic poles mainly seen for ascending arcs. The nature of these errors is, however, rather random and therefore not the main reason for the quality differences in Fig. 1.

The bi-monthly solution for 2009 clearly shows the best quality, slightly worse qualities were obtained for the 2010 and 2012 bi-monthly solutions. The worst quality was achieved for 2011, where a clear degradation is observed over the entire spectrum. The long-term solution shows an improved quality with respect to the bi-monthly solutions only for degrees beyond 30 . Above degree 110 the long-term solution is dominated by omission errors. Within the spectral range of degrees 30-110 the differences between the solutions are largely dominated by noise, but still the reduction of 
the differences lacks behind the statistical expectation (factor 3.2 instead of 3.9) when assuming the same noise behavior as for the outstanding November-December period of 2009. For degrees below 30 only marginal improvements are observed. This is a first indication that the low-degree differences with respect to ITG-GRACE2010 are dominated by systematic errors, which prevent an improvement by accumulating more data. As a further commonality we mention that degree 2 is not of a very good quality for all displayed solutions.

Figure 2 confirms the systematic nature of the low-degree differences by showing geoid height differences of the four bi-monthly solutions with respect to ITG-GRACE2010. A Gaussian filter with a radius of $300 \mathrm{~km}$ is adopted to focus on the long- to medium-wavelength part of the differences. Figure 2 clearly reveals that all four bi-monthly solutions are prone to severe systematic errors centered along the geo- magnetic equator. Barely visible in 2009, the size of the systematic errors is increasing over the years with a maximum impact on the bi-monthly solution from 2011, where maximum geoid height differences reach peak values of $20 \mathrm{~cm}$. Due to their systematic nature the errors are not reduced by accumulating longer data series but become more pronounced and are prominently seen also in the long-term solution shown in Fig. 3 (left). As they are still observed in combination models, e.g., in the Release 4 model of the official timewise solution where gradiometer data impact the solution already beyond degree 12 [Fig. 3 (right), Brockmann et al. (2013)], their nature is further analyzed in Sect. 4 and an approach is presented to significantly reduce them.

Figures 2 and 3 also exclude $C_{20}$ to reveal subtle differences such as the misfit of the sectorial coefficients of degree 2 with the reference solution. This misfit is in particular due
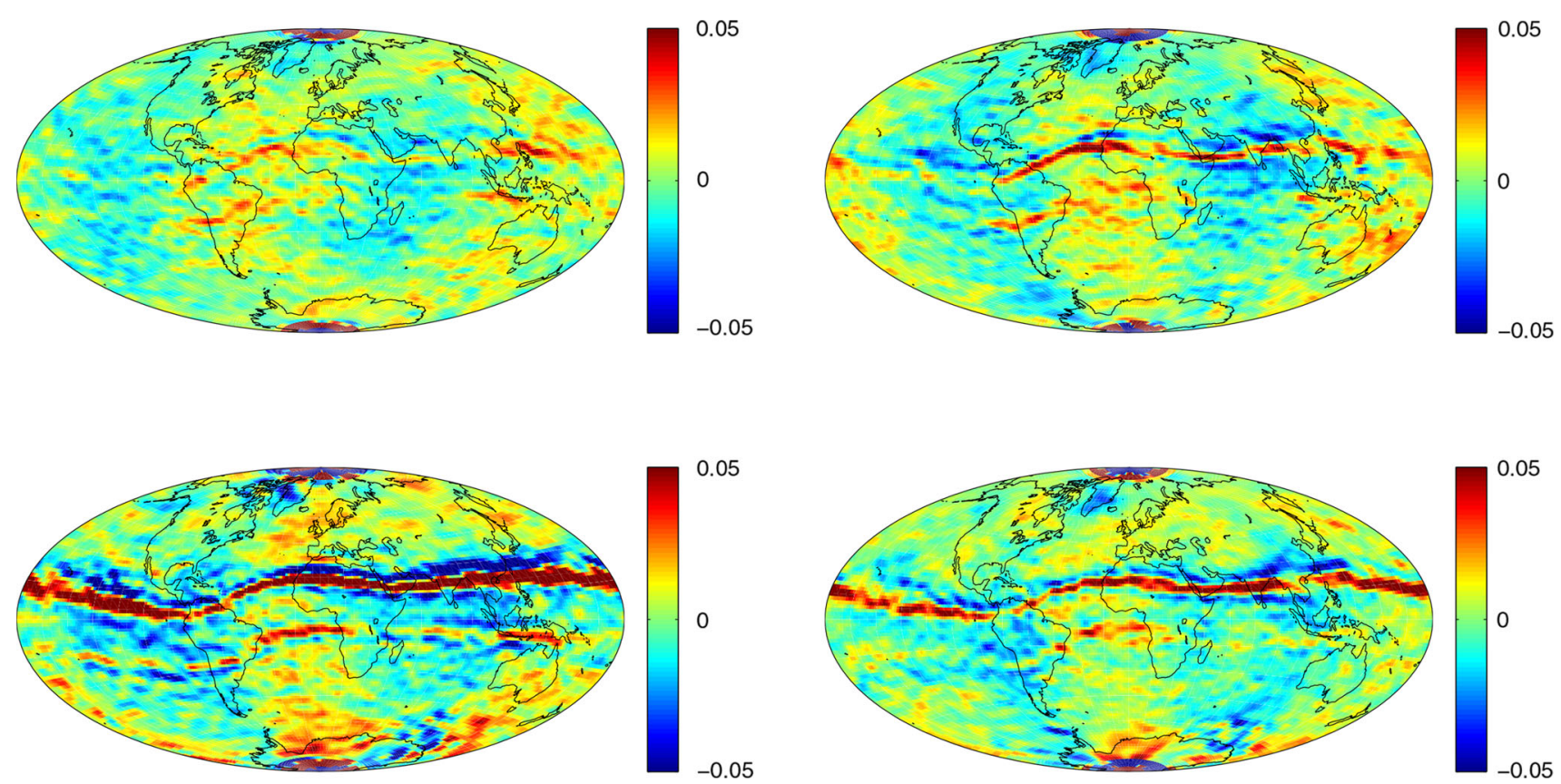

Fig. 2 Filtered geoid height differences (m) of bi-monthly gravity field solutions based on the kinematic positions of the PSO product with respect to ITG-GRACE2010 for the November-December period of 2009 (top left), 2010 (top right), 2011 (bottom left), 2012 (bottom right)
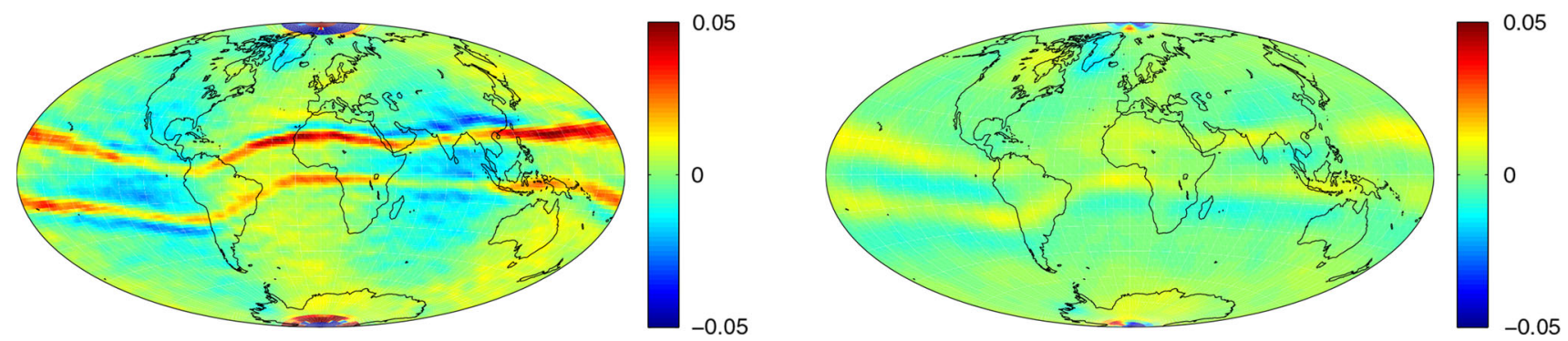

Fig. 3 Filtered geoid height differences (m) of the GPS-only long-term solution (left) and the Release 4 model of the timewise solution (right) with respect to ITG-GRACE2010 
to the coefficient $S_{22}$ and will be studied in the following subsection.

\subsection{Impact of accelerometer data and constraining}

The pseudo-stochastic pulses underlying the gravity field solutions presented so far are estimated as free parameters to generate solutions which have full flexibility to absorb model deficiencies. Subsequently such a solution is denoted as type (1). The adopted constraints are only weak (to just remove the singularity with the constant accelerations acting over the entire arc) and do not significantly influence the resulting gravity field solution. According to Sect. 2.1 either more realistic (absolute) constraints based on the accelerometer data may be adopted, which is subsequently denoted as a solution of type (2), or both, binned accelerometer data and more realistic constraints (absolute and relative) may be adopted, which is labelled as a solution of type (3).

Figure 4 (left) compares the impact of the improved constraining and the inclusion of accelerometer data on the actual differences of bi-monthly solutions covering the period of November-December 2009 with respect to ITGGRACE2010, as well as on the formal errors. The formal errors of solution (1) may be improved up to about degree 10 , indicating that the free estimation of pulses every $6 \mathrm{~min}$ overparametrizes the gravity field recovery. The differences in the formal errors between solution (2) and solution (3) are only minor with a slight preference for solution (3) due to the use of additional relative constraints. Figure 4 (left) also shows that the impact of different constrainings and the use of the accelerometer data on the actual solution are essentially confined to degree 2 . The level of the differences is obviously too large to profit from the improved constraining up to about degree 10 as promised by the formal errors.
The large discrepancy between formal errors and actual differences with respect to superior gravity field models based on GRACE K-Band data is not specific for GOCE but was already observed for gravity field recovery from kinematic positions of other LEO missions when not taking empirically derived covariance information into account (Jäggi et al. 2011b). As we will subsequently see, the determination of the low-degree terms is highly susceptible to systematic errors, which eventually might be responsible for the discrepancies in Fig. 4 (left).

Figure 5 shows geoid height differences of solutions (1) and (3) with respect to ITG-GRACE2010. A Gaussian filter with a radius of $300 \mathrm{~km}$ was again adopted to focus on the long- to medium-wavelength part of the differences. Figure 5 shows that the systematic errors centered along the geomagnetic equator are still present, but they can be reduced at least to a small extent by adopting a realistic constraining and by using accelerometer data. Moreover, the deterioration with respect to the sectorial coefficients of degree 2 could be removed due to the much improved quality of the $S_{22}$ coefficient.

In order to exclude that the quality of the low-degree coefficients is limited by unmodeled high-frequent timevariable effects of the Earth's gravity field, the atmospheric and oceanic dealiasing products (Flechtner et al. 2006) were in addition taken into account in the background modeling when estimating bi-monthly gravity field solutions of type (3). Figure 4 (right) compares the differences of solution (3) from Fig. 4 (left) to the difference when using and omitting AOD, respectively. With the exception of degree 2 the impact of the neglected AOD product is at least half a magnitude smaller than the differences with respect to ITGGRACE2010. In view of the quality of kinematic positions, and as opposed to ultra-precise K-Band observations, one
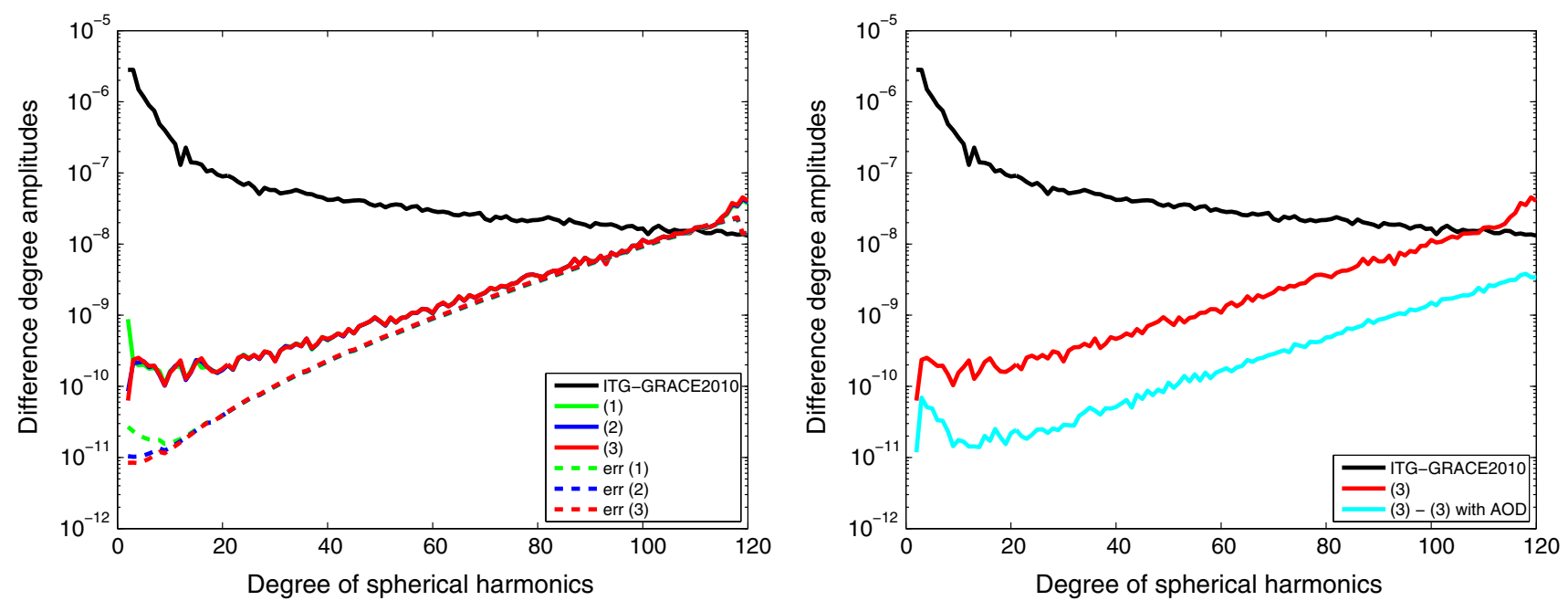

Fig. 4 Impact of constraining and accelerometer data (left) and AOD products (right) on gravity field recoveries from GOCE kinematic positions covering the November-December period of 2009 (zonal and near-zonal coefficients excluded) 

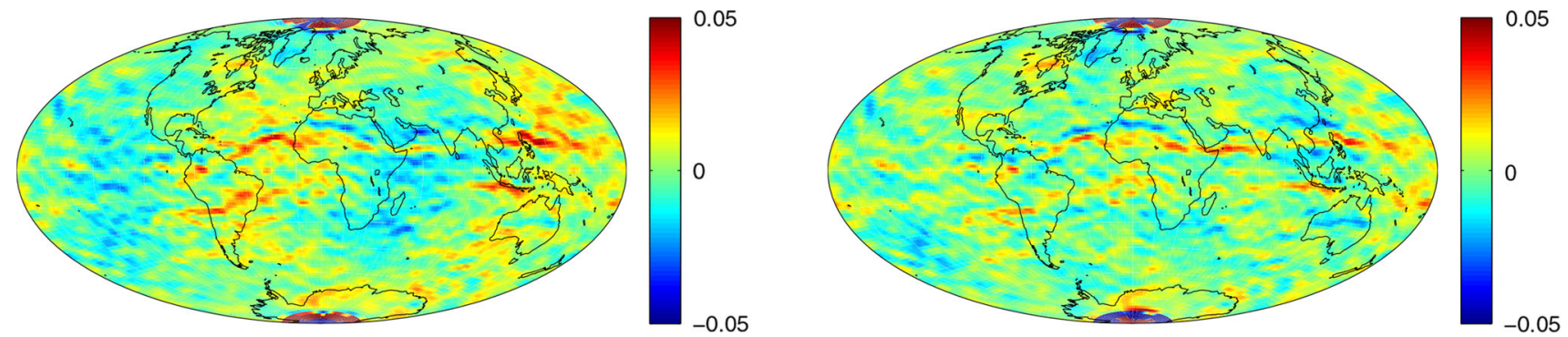

Fig. 5 Filtered geoid height differences (m) of bi-monthly solutions with respect to ITG-GRACE2010 for the November-December period of 2009 without (left) and with (right) accelerometer data
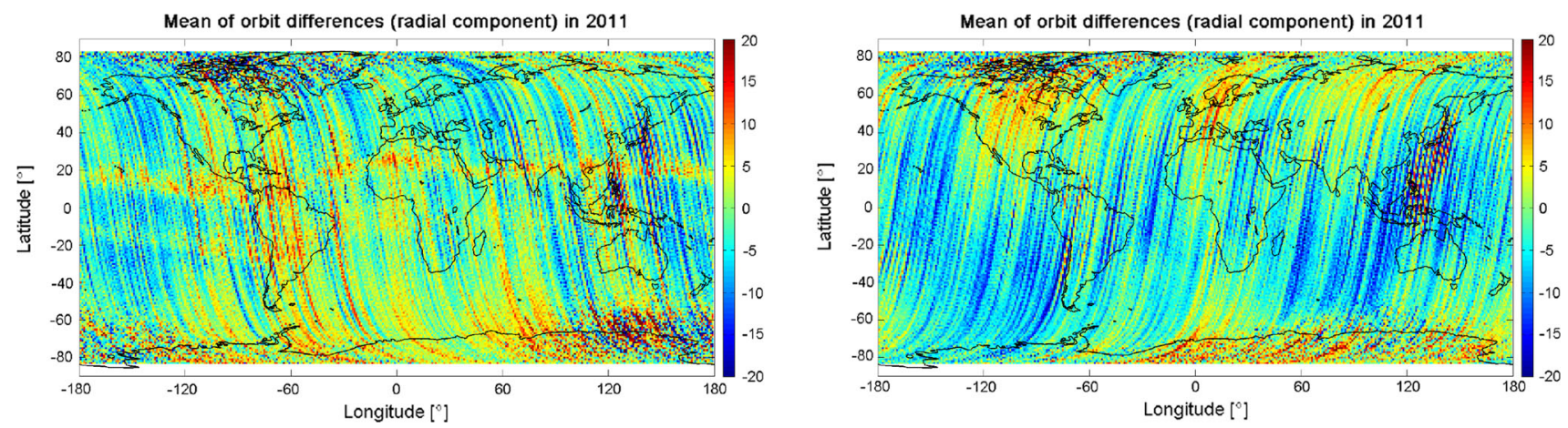

Fig. 6 Mean differences $(\mathrm{mm})$ per $1^{\circ} \times 1^{\circ}$ geographical bins between kinematic and reduced-dynamic orbits of the PSO product for ascending (left) and descending (right) arcs of the year 2011

may conclude that the neglect of AOD products does not pose a serious limitation to gravity field recovery from kinematic positions. The apparently different behavior of degree 2 in Fig. 4 (right) is simply due to the use of the rule of thumb by Gelderen and Koop (1997), which excludes $C_{20}$ when computing the differences. When also taking the zonal and near-zonal coefficients into account, the differences are still about half a magnitude smaller than the differences with respect to ITG-GRACE2010 for all degrees, but for degree 2 the behavior is indeed different. The inclusion of $C_{20}$ renders the differences to be on the same order as the differences with respect to ITG-GRACE2010. The neglect of AOD impacts the solutions from Fig. 4 (left) at degree 2, but is not capable to reduce the low-degree discrepancy to ITG-GRACE2010. Whether unmodeled time-variable signals related to land hydrology are a limiting factor will be assessed later on in this study.

\section{Analysis and removal of systematic effects around the geomagnetic equator}

In order to further study the systematic errors in Figs. 2 and 5, we first analyze the differences between the kinematic and reduced-dynamic orbits of the PSO product for the year 2011 in terms of mean values per geographical $1^{\circ} \times 1^{\circ}$ bins. As no constraints are imposed by dynamic models on kinematic positions, kinematic orbits are expected to be more affected by systematic errors than reduced-dynamic orbits (e.g., Jäggi et al. 2009). Apart from common signatures for ascending and descending arcs, e.g., larger mean differences over the geomagnetic poles, Fig. 6 clearly reveals differences between both orbit types along the geomagnetic equator, which occur for ascending arcs. With a size of up to $1 \mathrm{~cm}$ the effect is detectable thanks to the excellent quality of the GOCE PSO product (Bock et al. 2014). The signature is barely visible during the early phase of the mission (not shown), but gets more pronounced during recent years and is obviously responsible for the deterioration of the long-wavelength gravity field recovery shown in Sect. 3.

\subsection{Analysis of residuals}

In order to better confine the origin for the systematic errors around the geomagnetic equator we inspect the ionospherefree GPS carrier phase residuals of the determination of the reduced-dynamic PSO. As the effect might be related to the ionosphere, we average the residuals not at the GOCE subsatellite point but at the ionosphere piercing point of the corresponding observation directions. The ionosphere single layer is assumed to be at a height of $450 \mathrm{~km}$ for this experiment. Figure 7 shows these averaged residuals for 121 days (days 245-365) of the years 2009, 2010, and 2011, respectively. The systematic errors are clearly visible in the year 
Mean residuals at ionosphere-crossing: 2009, doys 245-365 $\times 10^{-3}$

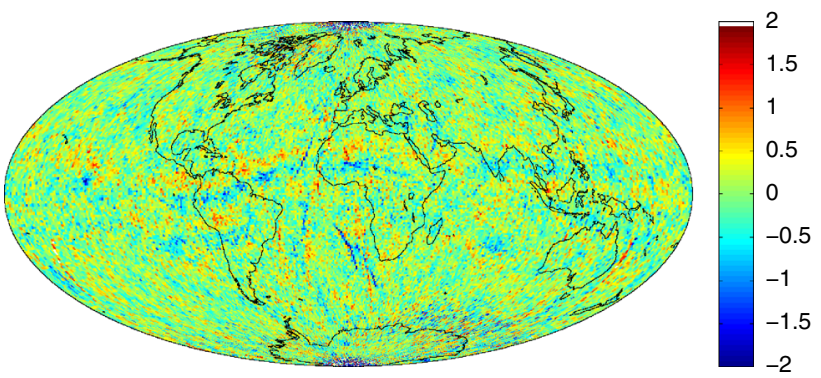

Mean residuals at ionosphere-crossing: 2011, doys 245-365

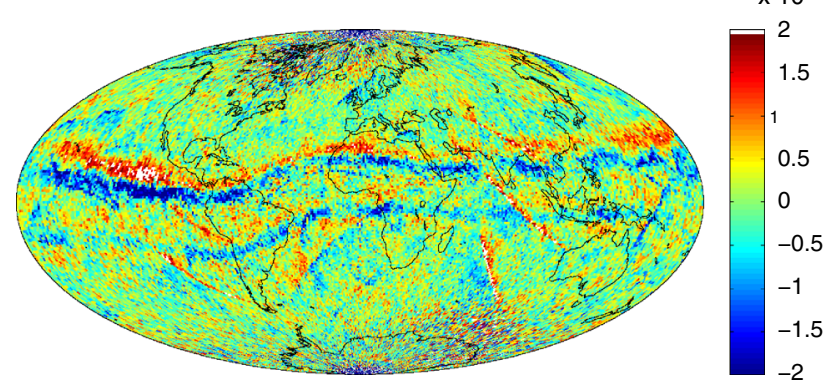

Mean residuals at ionosphere-crossing: 2010 , doys $245-365$

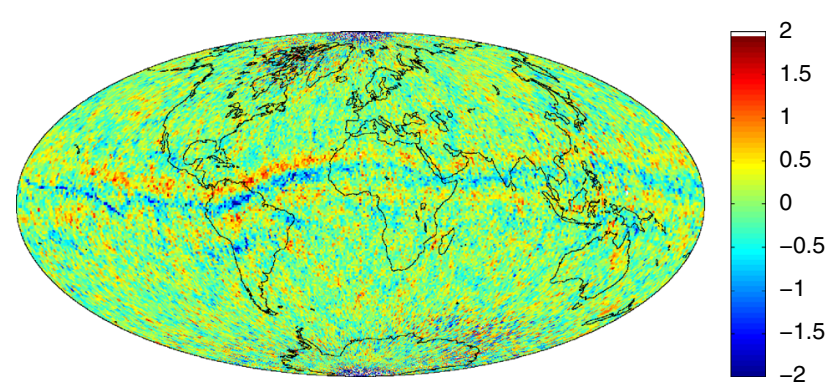

With HOI corrections, 300-365, 2011

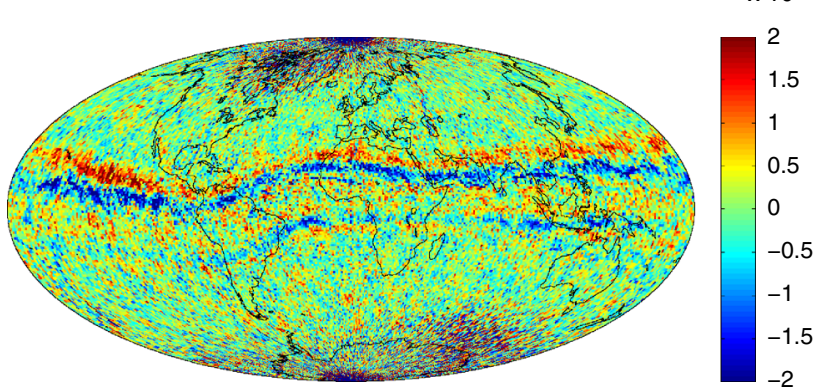

Fig. 7 Mean (m) of phase observation residuals mapped to the ionosphere piercing point, days 245-365, 2009 (top left), 2010 (top right), 2011 (bottom left), 2011 with HOI corrections applied (bottom right)

2011 but also for the earlier 2 years they are noticeable. As it is a systematic effect it might be a deficiency in the GPS observation model.

Because the systematics increase with increasing ionospheric density the higher order ionospheric (HOI) terms, which are not modeled in the operational GOCE processing (EGG-C 2010a), might cause the problem. We apply the HOI corrections for a short time interval in the GOCE orbit processing to check whether the systematics can be reduced or even be eliminated by this measure. Days 300365 in 2011 were selected for this purpose, because of the large effect during this time interval. The global ionosphere maps (GIMs, Schaer 1999) from the CODE (Center for Orbit Determination in Europe) analysis center were used to derive the total electron content (TEC) needed to compute the HOI correction terms. Figure 7 (bottom right) shows the phase observation residuals for the 66 days including HOI corrections mapped to the ionosphere piercing points. The data set is not as long as for the other maps shown in Fig. 7, only 66 instead of 121 days were used. Nevertheless, the systematics are still clearly visible, which implies that they are not caused by the HOI terms or, more precisely, that they can not be eliminated or significantly reduced by the current HOI model implementation. The HOI correction models as recommended by the IERS Conventions 2010 (Petit and Luzum 2010) were derived for terrestrial applications and therefore might not be suitable for the correction of LEO data (N. Jakowski, private communication). GOCE is flying in a very special orbit just below the assumed single layer of the ionosphere model. The orbit is essentially stationary (Sun-synchronous orbit) with respect to the ionosphere, and ascending arcs always pass through the region of highest electron density (dusk-dawn orbit). Therefore, only ascending but not descending arcs are affected by the systematic errors.

The systematic effects from Fig. 7 might also be related to short-term variations in the ionosphere such as scintillations (Jakowski et al. 2006). The geometry-free linear combination of GPS carrier phase observations is particularly well suited to detect short-term variations in the ionosphere because it only contains the ionospheric delay and the initial carrier phase ambiguities. For each tracked GPS satellite the linear combination

$$
\begin{aligned}
\Delta L_{4} & =L_{1}\left(t_{i}\right)-L_{2}\left(t_{i}\right)-\left(L_{1}\left(t_{i+1}\right)-L_{2}\left(t_{i+1}\right)\right), \\
i & =1, \ldots, n-1
\end{aligned}
$$

may be evaluated, where $\Delta L_{4}$ is the ionosphere change within the intervals $\Delta t=t_{i+1}-t_{i}, L_{1}$ and $L_{2}$ are the phase observations on the two carrier frequencies, $t_{i}$ is the time at observation epoch $i$ and $n$ is the number of $1 \mathrm{~Hz}$ observation epochs. In order to get rid of large short-term variations in the ionosphere, observations for which the absolute value of $\Delta L_{4}$ is larger than a certain threshold are excluded from the processing. Empirically, a threshold of $5 \mathrm{~cm} / \mathrm{s}$ was found to be suitable. 
The GOCE orbit determination has been repeated with this new, reduced set of ionosphere-free carrier phase GPS observations. The phase observation residuals of the reduced-dynamic orbit determination are again mapped to the ionosphere piercing point and averaged for the time span from days 300-365 in the year 2011 (Fig. 8). The systematic effect around the geomagnetic equator could obviously be removed to a large extent by excluding the observations with ionosphere changes larger than $5 \mathrm{~cm} / \mathrm{s}$. Figure 9 shows the percentage of remaining observations and resulting kinematic positions of this screened data set with respect to the original data. Despite the exclusion of all observations related to large ionosphere changes, this merely corresponds to $94.4 \%$ of the total set of available GPS observations. Nearly all of the removed observations are located at bands along the geomagnetic equators. On average $93.8 \%$ of the kinematic positions can still be determined, which implies a small reduction of about $6.2 \%$ for the set of kinematic positions used for gravity field recovery. For the time span of days 300-365 in the years 2009, 2010, and the 2012, the reduction is even significantly smaller (not shown), amounting to 0.1 , 0.2 , and, $3.7 \%$, respectively.

\subsection{Gravity field recovery using improved kinematic positions}

Gravity field recovery from Sect. 3 was repeated by replacing the kinematic positions from the official PSO solution

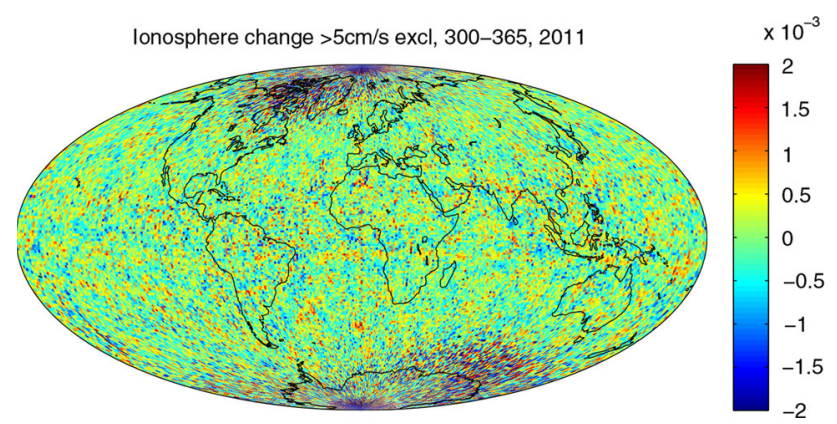

Fig. 8 Mean (m) of phase observation residuals mapped to the ionosphere piercing point, days 300-365, 2011; observations with $\left|\Delta L_{4}\right|>5 \mathrm{~cm} / \mathrm{s}$ are excluded

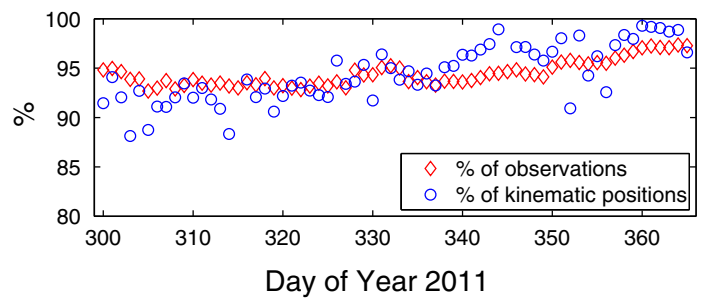

Fig. 9 Percentage of GPS ionosphere-free observations and kinematic positions with respect to the original data set for days 300-365, 2011 by the kinematic positions derived in Sect. 4.1. Figure 10 shows the square-roots of the degree difference variances of the new GPS-only solutions up to d/o 120 with respect to ITG-GRACE2010 in comparison with the solutions shown in Sect. 3. Only marginal differences are observed for the bi-monthly solution for 2009 due to the weak signature of the systematic errors along the geomagnetic equator. For the bi-monthly solutions of 2010, 2011, and 2012, however, a clear reduction of the differences with respect to ITG-GRACE2010 is observed over the entire spectrum. The strongest reduction occurs for 2011, where the strongest signature of the systematic errors along the geomagnetic equator is observed. Apart from 2011 the differences of all bimonthly solutions are on a very similar level. It has to be noted as well, however, that the very low degrees cannot be improved by using the new kinematic positions due to the smaller spatial scales of the systematic errors.

In order to confirm that the systematic errors related to the geomagnetic equator could be significantly reduced, Fig. 11 shows geoid height differences of the four bi-monthly solutions based on the new orbits with respect to ITGGRACE2010. A Gaussian filter with a radius of $300 \mathrm{~km}$ was adopted to focus on the long- to medium-wavelength part of the differences. A comparison with Fig. 2 shows that a large part of the signature could indeed be removed.

\section{Time variable signals from GPS-only solutions}

Based on CHAMP kinematic positions Weigelt et al. (2013) have recently shown that time variable gravity (TVG) signals with annual periods can be recovered from CHAMP monthly gravity field solutions by adopting Kalman filter techniques. In this section we assess the contribution (and limitation) of GOCE kinematic positions to derive TVG signals by simple means, and whether their neglect in Sect. 3 is responsible for the limited quality of the low-degree coefficients. Due to the generally limited sensitivity of TVG recovery from kinematic positions, the longest possible data span, covering the time period between November 1, 2009 and October 20, 2013, is used to compare two straightforward methods to solve for TVG signals up to d/o 10 .

As a consequence of the GOCE orbital characteristics the ground-tracks have a repeat cycle of 61 days with a sub-cycle of 20 days. As opposed to the GRACE mission, where data are typically processed in monthly batches to achieve a full coverage of the Earth, either 20, 40, or 60 days are needed for GOCE. Due to the very low resolution anticipated by $\mathrm{d} / \mathrm{o}$ 10 we derive solutions every 20 days in Sect. 5.1. Similar to the findings of Prange (2010) for CHAMP, "raw" 20-day solutions are governed by large noise, even if omission errors beyond d/o 10 are reduced by high-quality static gravity field models. Therefore we confine ourselves in Sect. 5.1 to repre- 

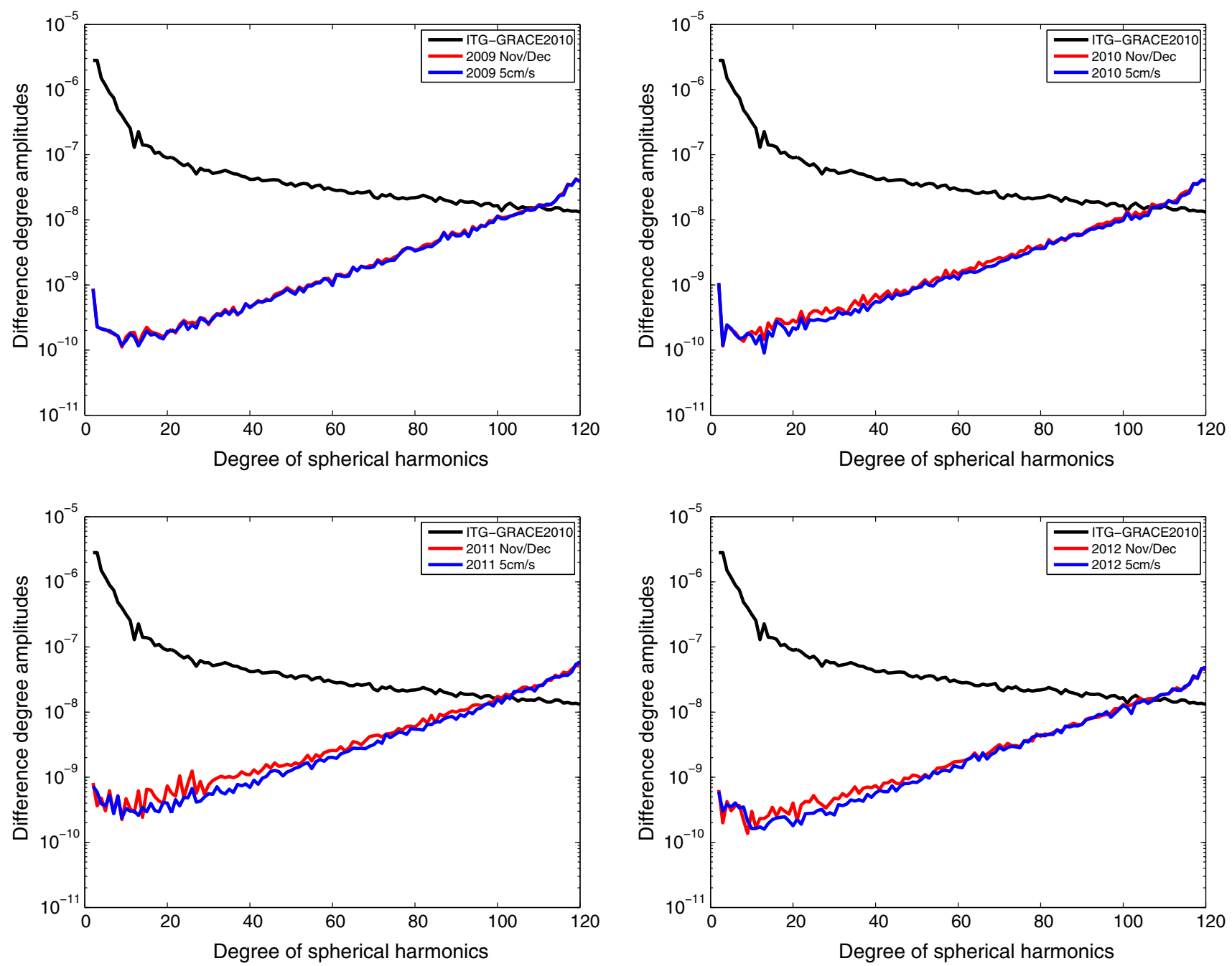

Fig. 10 Square-roots of degree difference variances of gravity field recoveries from GOCE kinematic positions (zonal and near-zonal coefficients excluded) based on GPS observations with ionosphere change $<5 \mathrm{~cm} / \mathrm{s}$ for different time periods

sent the 20-day solutions a posteriori by an annual signal. It has to be emphasized that this is a representation of the mean TVG signal only.

Due to frequent gradiometer calibrations and several anomalies limiting the availability of the GOCE data, the analyzed time period between Nov 1, 2009 and Oct 20, 2013 cannot be completely covered with 20-day solutions. In Sect. 5.2 we directly solve for the annual signal using the original NEQs. This makes the intermediate and somewhat cumbersome derivation of 20-day solutions obsolete and offers a more rigorous estimation of the annual signals from a statistical point of view.

\subsection{Fitting annual signals}

The sensitivity of orbital positions to TVG signals is restricted to the long-wavelength part of the Earth's gravity field. It is therefore important to reduce omission errors to the extent possible, either by simultaneously estimating the static gravity field beyond a certain cut-off degree, or by introducing the static part from a previously established or external solution. For the latter approach two types of solutions are produced. Either a state-of-the-art static gravity field model based on different tracking techniques is used such as GOCO03S (Mayer-Gürr et al. 2012), or the GOCE long-term GPS-only solution from Sect. 3.

In order to assess whether the 20-day GOCE solutions contain information on the most dominant TVG signals, an annual signal is fitted through the individual 20-day solutions. Let us assume that the SH coefficients valid for a certain 20-day interval $\left[t_{i}-10 d, t_{i}+10 d\right]$ are denoted by $\mathbf{x}_{i}$. Each SH coefficient $x_{i, k} \in \mathbf{x}_{i}$ is then represented as

$$
\begin{aligned}
x_{i, k}+v_{i, k}= & a_{k} \cdot \cos \left(\omega\left(t_{i}-t_{0}\right)\right) \\
& +b_{k} \cdot \sin \left(\omega\left(t_{i}-t_{0}\right)+c_{k},\right.
\end{aligned}
$$

where $\omega$ denotes the annual frequency, which is represented by a cosine and sine term with amplitudes $a_{k}$ and $b_{k}$, respec- 

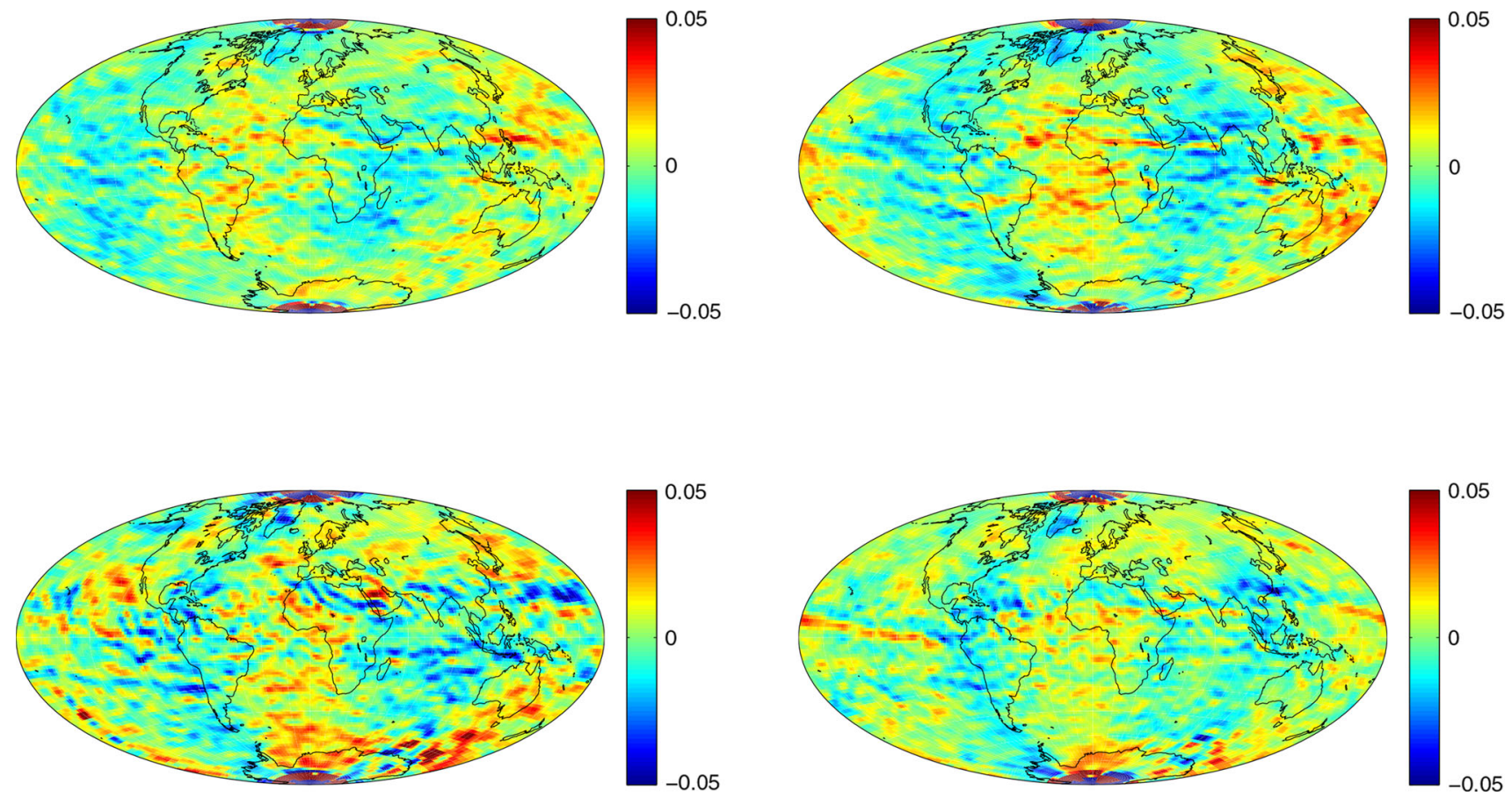

Fig. 11 Filtered geoid height differences $(\mathrm{m})$ of bi-monthly gravity field solutions based on kinematic positions using GPS observations with ionosphere change $<5 \mathrm{~cm} / \mathrm{s}$ with respect to ITG-GRACE2010 for the November-December period of 2009 (top left), 2010 (top right), 2011 (bottom left), 2012 (bottom right)

tively. $t_{0}$ represents an arbitrarily selectable reference epoch, $c_{k}$ the offset, and $v_{i, k}$ the residuals, which are minimized in the least-squares sense by fitting each coefficient individually and by using the formal errors of the 20-day solutions for an appropriate weighting. Correlations between the $\mathrm{SH}$ coefficients are thus neglected and care has to be taken that the underlying 20-day solutions are homogenously covered by daily arcs, in such a way that the estimates $\mathbf{x}_{i}$ properly refer to the center $t_{i}$ of the time interval.

A total of sixty-two, 20-day solutions were generated up to $\mathrm{d} / \mathrm{o} 10$ by using the two static gravity field models GOCO03S and the GOCE GPS-only solution from Sect. 3 beyond degree 10, respectively. The large number of solutions provides a good sampling of the annual signal over several periods (3.97 years), and discretization effects due to the use of 20-day intervals may still be kept small and do not induce significant signal damping when recovering the signal by a posteriori fitting the simple model from Eq. (5). Figure 12 (top row) shows a visual comparison in terms of geoid heights of the annual amplitude of the sine signal, which is the dominant term in Eq. (5) when identifying $t_{0}$ with the beginning of a year (Tapley et al. 2004). A Gaussian filter with a radius of $1,500 \mathrm{~km}$ was applied for all solutions to focus on the long-wavelength part of the signal. The general structure of the two solutions is similar, confirming that only the largest part of the signal may be captured. Although the detailed shape of the signal over land and the artefacts over the oceans are slightly different, the use of a state-of-the- art gravity field background model does not seem to provide significant advantage with respect to the GOCE GPS-only solution. The degraded quality of the zonal and near-zonal coefficients of the GOCE GPS-only solution does not seem to seriously degrade the recovery of the low-degree annual time variable signal.

\subsection{Direct approach}

A more rigorous estimation of annual signals may be achieved by directly using the NEQ systems established in Sect. 3. Let us assume that the NEQ system associated with the kinematic positions from daily arc $i$ reads as

$\mathbf{N}_{i} \mathbf{x}=\mathbf{b}_{i}$,

where $\mathbf{x}$ denotes the unknown SH coefficients. $\mathbf{N}_{i}$ and $\mathbf{b}_{i}$ denote the contributions of this particular arc to the total normal equation matrix and right-hand side, which would be obtained by accumulating the contributions from all individual arcs.

In analogy to Eq. (5) a particular SH coefficient $x_{k}$ shall be related to an annual signal according to

$x_{k}=a_{k} \cdot \cos \left(\omega\left(t_{i}-t_{0}\right)\right)+b_{k} \cdot \sin \left(\omega\left(t_{i}-t_{0}\right)\right)+c_{k}$.

In matrix form this relation may be written as a linear transformation between the new parameters $\tilde{\mathbf{x}}$ and the original 

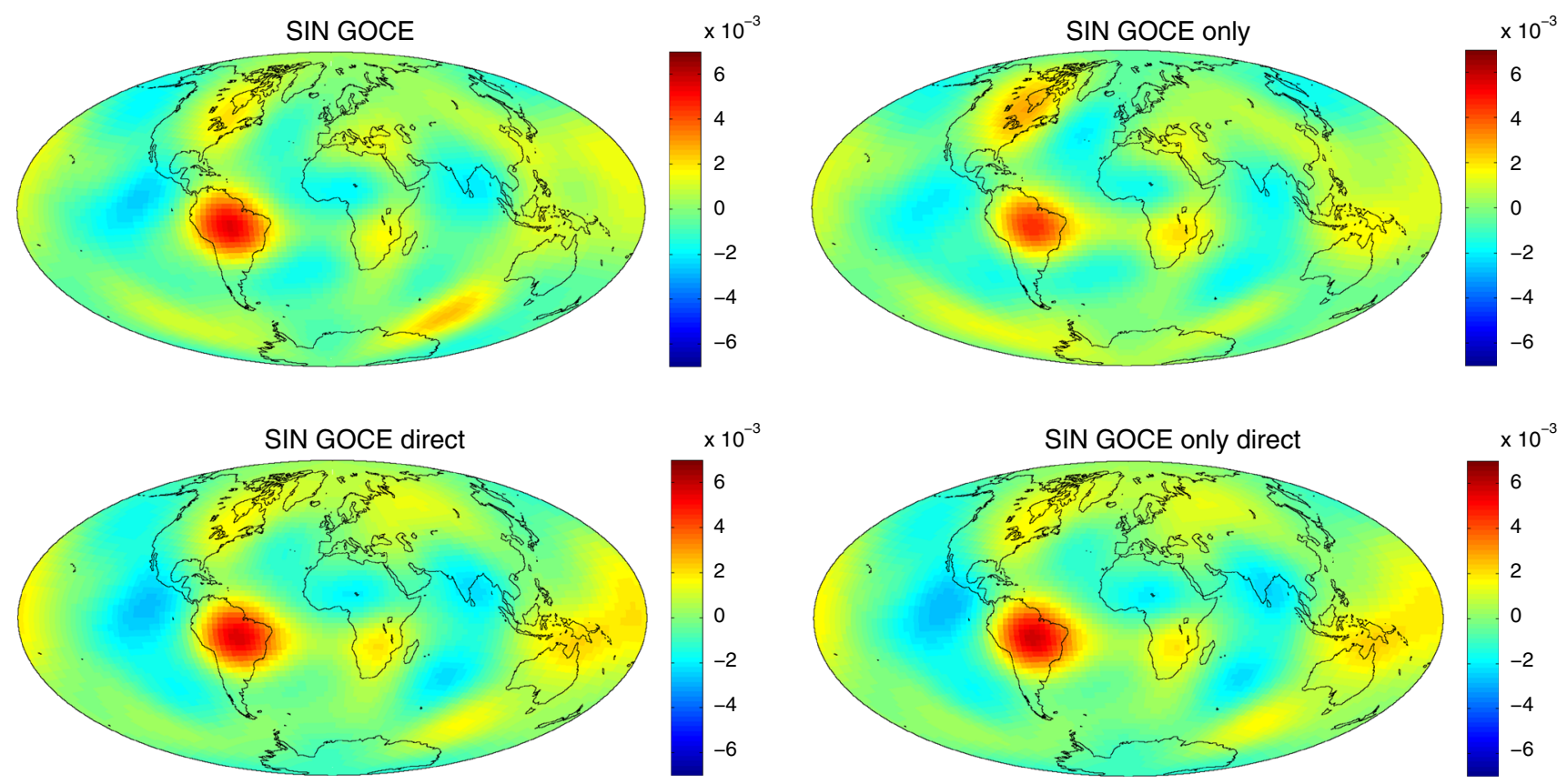

Fig. 12 Annual amplitude (m) of the sine term when using GOCO03S (left column) and the GOCE GPS-only solution (right column) beyond degree 10 (top line: a posteriori fit, bottom line: direct approach)

SH coefficients $\mathbf{x}$ as

$\mathbf{x}=\mathbf{B}_{i} \tilde{\mathbf{x}}+\mathbf{d B}_{i}$,

where the original and new parameter vectors are defined as

$\mathbf{x}=\left(\begin{array}{c}x_{1} \\ \vdots \\ x_{n}\end{array}\right), \quad \tilde{\mathbf{x}}=\left(\begin{array}{c}a_{1} \\ b_{1} \\ c_{1} \\ \vdots \\ a_{n} \\ b_{n} \\ c_{n}\end{array}\right)$,

$n$ being the number of SH coefficients. The transformation matrix reads as

$\mathbf{B}_{i}$

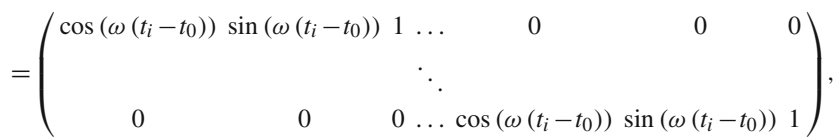

whereas $\mathbf{d B}_{i}=\mathbf{0}$ holds for the offset vector. According to parameter transformation in least-squares adjustment (e.g., Brockmann 1997), the NEQ matrix and right-hand side referring to the transformed parameters $\tilde{\mathbf{x}}$ read as

$\tilde{\mathbf{N}}_{i} \tilde{\mathbf{x}}=\tilde{\mathbf{b}}_{i}$

with

$\tilde{\mathbf{N}}_{i}=\mathbf{B}_{i}^{\prime} \mathbf{N}_{i} \mathbf{B}_{i}, \quad \tilde{\mathbf{b}}_{i}=\mathbf{B}_{i}^{\prime}\left(\mathbf{b}_{i}-\mathbf{N}_{i} \mathbf{d B}_{i}\right)$
The already existing NEQ contributions $\mathbf{N}_{i}$ and $\mathbf{b}_{i}$ from each daily arc may thus be used and transformed according to Eq. (12) to directly solve for the annual signal. The total NEQ system is obtained by accumulating all individual contributions (11) from each arc, which automatically ensures that correlations between coefficients are correctly taken into account. As opposed to the a posteriori fitting of the annual signal in Sect. 5.1, potential discretization effects are not an issue and the somewhat cumbersome processing of 20-day solutions is superfluous.

Figure 12 (bottom row) visually compares the annual amplitude of the sine term when directly solving for the annual amplitudes based on GOCO03S and the GOCE GPS-only long-term solution from Sect. 3, respectively. As opposed to the a posteriori fit of the annual signal from 20day solutions, more consistent results are obtained, visually not revealing any significant differences caused by the use of the different background models. In order to better quantify the differences Fig. 13 shows the TVG signal in terms of square-roots of degree variances. Very consistent solutions are obtained by the direct approach with only a small dependency on the static background model. Slightly less consistent solutions are observed for the a posteriori fit. Also, the recovered annual signal is different compared to the direct approach. The RMS values of geoid height differences with respect to a GRACE solution based on the sine component (see next section) read as $0.86 \mathrm{~mm}$ and $0.82 \mathrm{~mm}$ for the a posteriori fit and the direct approach, respectively. For the cosine component the RMS values are $1.7 \mathrm{~mm}$ and $1.5 \mathrm{~mm}$, indicating a small preference for the direct approach. 

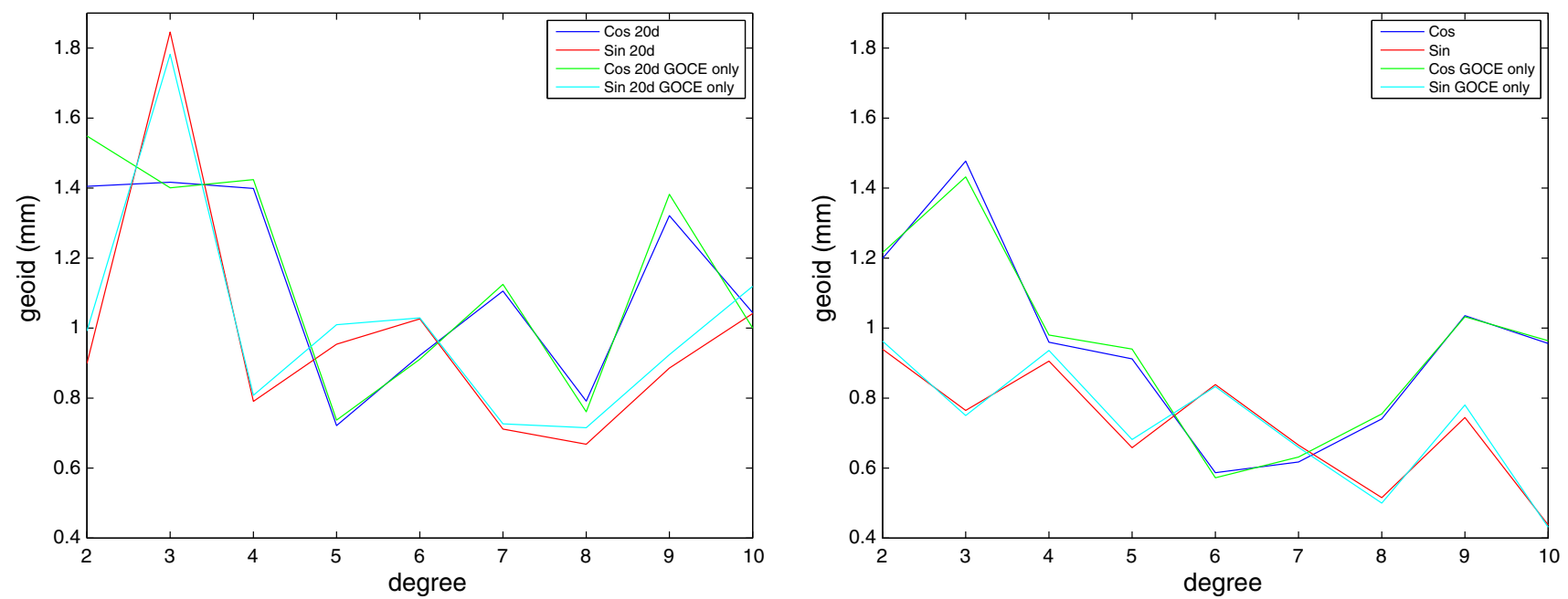

Fig. 13 Square-roots of degree variances of a posteriori (left) and direct (right) recoveries of the annual TVG signal from GOCE kinematic positions using different static gravity field background models beyond d/o 10

It was checked that the direct estimation of TVG signals is not able to significantly change the co-estimated low-degree coefficients of the static part of the gravity field (coefficients $c_{k}$ in Eq. (7), not shown). Unmodeled TVG signals related to land hydrology are thus not responsible for the limited quality of the low-degree coefficients reported in Sect. 3.

\subsection{Comparison with CHAMP and GRACE}

Similar to the gravity mission CHAMP, GPS hl-SST data from GOCE is not primarily intended to recover TVG signals. A comparison of CHAMP- and GOCE-based results with TVG signals extracted from GRACE indicates, however, what can be expected from GPS hl-SST data. Figure 14 visually compares the annual amplitude of the sine and cosine terms recovered from GOCE GPS data (a posteriori fit of the solution from Sect. 5.1 using GOCO03), CHAMP GPS data (Weigelt et al. 2013), and GRACE K-Band data (Meyer et al. 2012). In order to be compatible with the GPS-based solution, the CHAMP and GRACE monthly solutions were in analogy fitted by an annual signal and a Gaussian filter with a radius of $1500 \mathrm{~km}$ was applied. The general structure of the sine term is similar for all solutions, but details are quite different for the two GPS-based solutions. The RMS values of geoid height differences with respect to the GRACE solution are 0.86 and $0.67 \mathrm{~mm}$ for GOCE and CHAMP, respectively. Much larger differences are observed for the cosine term. The GOCE solution fails to a large extent to correctly assign the phase to the annual signal. The RMS values of the geoid height differences with respect to the GRACE solution are 1.70 and $0.72 \mathrm{~mm}$ for GOCE and CHAMP, respectively. The recovery thus seems to be much better for CHAMP, but it has to be emphasized that the CHAMP solution is based on a longer time series and stems from a dedicated processing based on a Kalman filter approach (Weigelt et al. 2013). A reduction of the CHAMP time series to the same length as the GOCE series would not significantly change the quality of the recovered TVG signals (not shown). The adoption of a Kalman filter approach to the 20-day GOCE solutions, however, would indeed significantly reduce the cosine amplitudes as well (M. Weigelt, private communication), yielding a solution with an RMS of geoid height differences of $0.82 \mathrm{~mm}$ for the sine and $1.00 \mathrm{~mm}$ for the cosine component, which is in-between the CHAMP and GOCE results based on the Kalman filter and the straightforward approach, respectively. Possible reasons for the inferior quality of the GOCE cosine term are discussed by Visser et al. (2014).

\section{Summary}

GOCE GPS-only gravity field determination based on the kinematic positions of the official GOCE PSO product was performed using the Celestial Mechanics Approach. A longterm solution based on data from November 1, 2009 until July 31,2012 , and solutions based on bi-monthly periods covering November-December of the years 2009, 2010, 2011, and 2012, were generated. All solutions revealed to be affected by severe systematic errors centered along the geomagnetic equator. Barely visible in 2009, the size of the systematic errors increased over the years with a maximum impact on the bi-monthly solution from 2011. Based on efficient methods to take into account accelerometer data for already existing normal equations which were generated without accelerometer data, a positive impact of the improved modeling of non-gravitational accelerations can be demonstrated, but the effect is confined to the coefficients of degree two. Essen- 

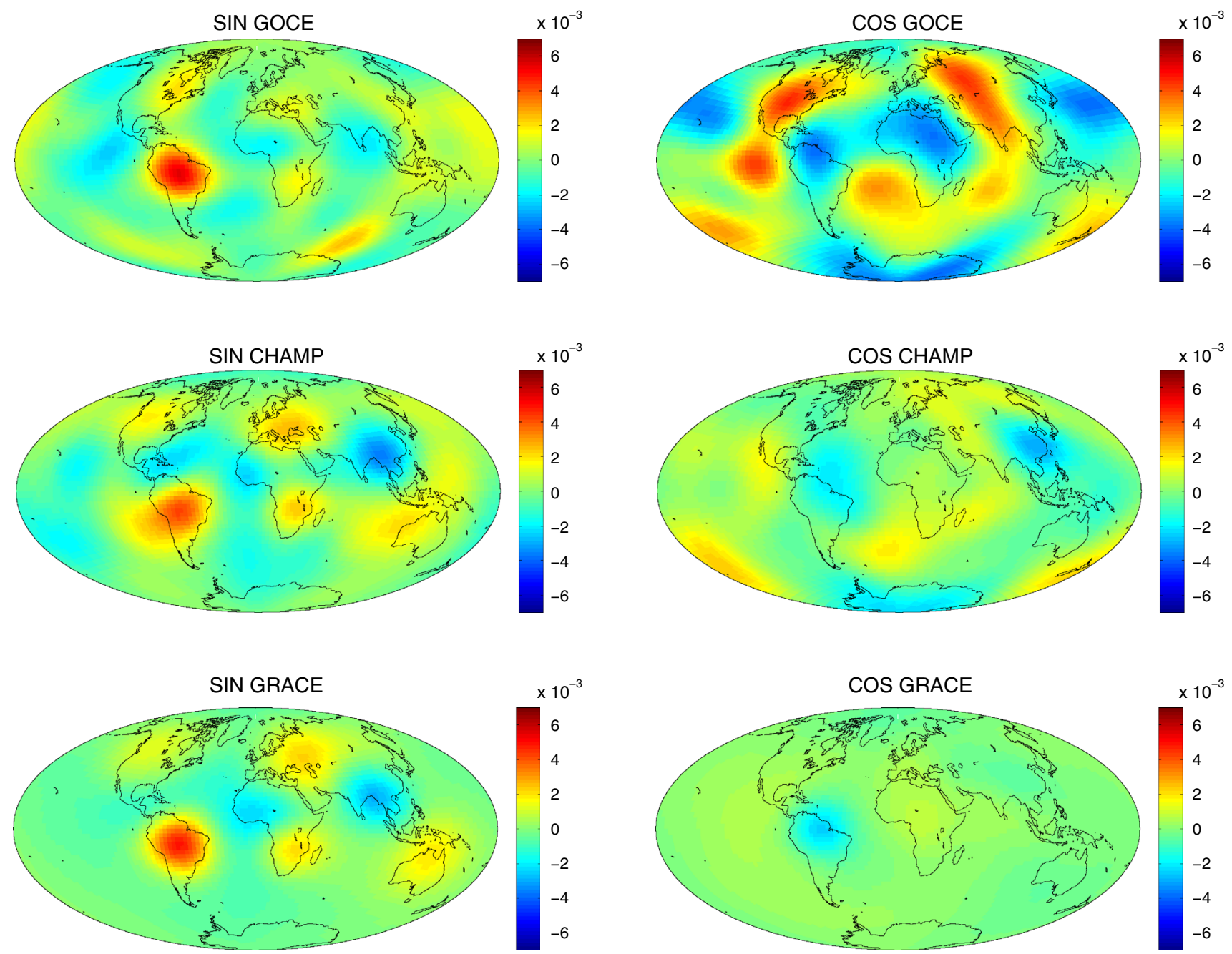

Fig. 14 Annual amplitude (m) of the sine (left column) and cosine (right column) term recovered from GOCE GPS data (top row), CHAMP GPS data (middle row) and GRACE K-Band data (bottom row)

tially the same conclusion may be drawn when modeling high-frequency time-variable effects of the Earth's gravity field.

The pronounced systematic errors along the geomagnetic equator may be traced back to the kinematic positions of the PSO product and eventually to the ionosphere-free GPS carrier phase observations by averaging the residuals at the ionosphere piercing point of the corresponding observation directions. As the systematic errors increase with increasing ionosphere density, the unmodeled higher order ionospheric (HOI) terms in the orbit determination process according to the GOCE standards were suspected to be responsible for the error pattern. However, no significant reduction could be seen by adopting the HOI correction model as recommended by the IERS Conventions 2010. As the HOI correction model was originally derived for terrestrial applications, further investigations are needed to see whether a model extension is needed for LEO data. Also the use of global ionosphere maps (GIMs) might not be optimal to extract the total electron content needed to compute the HOI correction terms, because GIMs do not represent short-term ionospheric variations. However, because the affected kinematic positions may be confined to situations with large ionopsheric changes, an empirical approach may be followed in the meantime. Discarding GPS data with ionospheric changes larger than $5 \mathrm{~cm} / \mathrm{s}$ leads to a small reduction of at maximum $6 \%$ of the useable set of kinematic positions for severe ionosphere conditions, but, more importantly, yields a strong reduction of the systematic errors in the gravity field recovery and a considerable improvement of the solutions between degrees 10 and 60 . This might be a suitable approach for a reprocessing of the GOCE PSO product if the physical cause of the systematic errors cannot be found or explained.

Eventually, the impact of unmodeled time variable gravity (TVG) signals related to land hydrology on GOCE GPSbased gravity field recovery was assessed using straightfor- 
ward approaches and the longest possible time interval from November 1, 2009 until October 20, 2013. Based on the direct estimation of an annual signal using already existing normal equations, which were generated without TVG parameters, or a simple a posteriori fit of the annual signal through 20-day solutions, a limited sensitivity of GOCE gravity field solutions to the largest annual signal related to land hydrology could be demonstrated when using kinematic positions. The comparison with results from CHAMP and GRACE indicates that more refined analysis techniques are needed to better exploit the TVG signal content from GOCE, but the presented methods are sufficient to decide that unmodeled TVG signals due to land hydrology are not responsible for the still limited quality of the low-degree coefficients below degree 10.

Acknowledgments This work was partly performed in the framework of the GOCE High-level Processing Facility (HPF), which is funded by ESA. The support of ESA is gratefully acknowledged. We also thank M. Weigelt for adopting the Kalman filter approach to the 20-day GOCE gravity field solutions.

\section{References}

Beutler G, Jäggi A, Mervart L, Meyer U (2010a) The celestial mechanics approach: theoretical foundations. J Geod 84(10):605-624. doi:10. 1007/s00190-010-0401-7

Beutler G, Jäggi A, Mervart L, Meyer U (2010b) The celestial mechanics approach: application to data of the GRACE mission. J Geod 84(11):661-681. doi:10.1007/s00190-010-0402-6

Baur O, Bock H, Höck E, Jäggi A, Krauss S, Mayer-Gürr T, Reubelt T, Siemes C, Zehentner N (2014) Comparison of GOCE-GPS gravity fields derived by different approaches. J Geod. doi:10.1007/ s00190-014-0736-6

Bock H, Jäggi A, Meyer U, Visser P, van den IJssel J, van Helleputte T, Heinze M, Hugentobler U (2011a) GPS-derived orbits for the GOCE satellite. J Geod 85(11):807-818. doi:10.1007/s00190-011-0484-9

Bock H, Jäggi A, Meyer U, Dach R, Beutler G (2011b) Impact of GPS antenna phase center variations on precise orbits of the GOCE satellite. Adv Space Res 47(11):1885-1893. doi:10.1016/j.asr.2011. 01.017

Bock H, Jäggi A, Beutler G, Meyer U (2014) GOCE: precise orbit determination for the entire mission. J Geod. doi:10.1007/ s00190-014-0742-8

Bouman J, Fiorot S, Fuchs M, Gruber T, Schrama E, Tscherning C, Veicherts M, Visser P (2011) GOCE gravitational gradients along the orbit. J Geod 85:791-805. doi:10.1007/s00190-011-0464-0

Brockmann E (1997) Combination of solutions for geodetic and geodynamic applications of the global positioning system (GPS). Geodätisch-geophysikalische Arbeiten in der Schweiz, vol 55. Schweizerische Geodätische Kommission

Brockmann JM, Höck E, Krasbutter I, Mayer-Gürr T, Pail R, Schuh WD, Zehentner N (2013) Performance of the fourth generation GOCE time-wise Earth gravity field model. Geophys Res Abstr 15

Cesare S (2008) Performance requirements and budgets for the gradiometric mission, 4th edn. Technical report, Thales Alenia Space

Dach R, Brockmann E, Schaer S, Beutler G, Meindl M, Prange L, Bock H, Jäggi A, Ostini L (2009) GNSS processing at CODE: status report. J Geod 83(3-4):353-366. doi:10.1007/s00190-008-0281-2
Drinkwater M, Haagmans R, Muzi D, Popescu A, Floberghagen R, Kern M, Fehringer M (2006) The GOCE gravity mission: ESA's first core explorer. In: 3rd GOCE User Workshop, 6-8 Nov 2006, Frascati, Italy, pp 1-7, ESA SP-627

European GOCE Gravity Consortium (EGG-C) (2010a) GOCE Standards, GO-TN-HPF-GS-0111, Issue 3.2

European GOCE Gravity Consortium (EGG-C) (2010b) GOCE Level 2 Product Data Handbook, GO-MA-HPF-GS-0110, Issue 4.3

ESA (2004) Swarm-The Earth's Magnetic Field and Environment Explorers. Reports for Mission Selection, The Six Candidate Earth Explorer Missions, ESA SP-1279(6)

ESA (2006) GOCE L1b Products User Handbook, GOCE-GSEGEOPG-TN-06-137, Issue 1.1

Flechtner F, Schmidt R, Meyer U (2006) De-aliasing of short-term atmospheric and oceanic mass variations for GRACE. In: Flury J, Rummel R, Reigber C, Rothacher M, Boedecker G, Schreiber U (eds) Observation of the earth system from space. Springer, Heidelberg, pp 83-97

Flechtner F, Morton P, Watkins M, Webb F (2013) Status of the GRACE follow-on mission. In: Proceedings of the international association of geodesy symposia gravity, geoid and height system (GGHS2012), 9-11 Oct 2012, Venice, Italy, IAGS-D-12-00141, accepted

Floberghagen R, Fehringer M, Lamarre D, Muzi D, Frommknecht B, Steiger C, Pineiro J, da Costa A (2011) Mission design, operation and exploitation of the gravity field and steady-state ocean circulation explorer mission. J Geod 85(11):749-758. doi:10.1007/ s00190-011-0498-3

Intelisano A, Mazzini L, Notarantonio A, Landenna S, Zin A, Scaciga L, Marradi L (2008) Recent flight experiences of TAS-I on-board navigation equipments. In: Proceedings of the 4th ESA workshop on satellite navigation user equipment technologies, NAVITEC2008, 10-12 Dec 2008, Noordwijk, The Netherlands

Jäggi A, Hugentobler U, Beutler G (2006) Pseudo-stochastic orbit modeling techniques for Low-Earth orbiters. J Geod 80(1):47-60. doi:10. 1007/s00190-006-0029-9

Jäggi A, Bock H, Pail R, Goiginger H (2008) Highly reduced dynamic orbits and their use for global gravity field recovery: a simulation study for GOCE. Stud Geophys Geod 52(3):341-359. doi:10.1007/ s11200-008-0025-z

Jäggi A, Dach R, Montenbruck O, Hugentobler U, Bock H, Beutler G (2009) Phase center modeling for LEO GPS receiver antennas and its impact on precise orbit determination. J Geod 83(12):1145-1162. doi:10.1007/s00190-009-0333-2

Jäggi A, Bock H, Prange L, Meyer U, Beutler G (2011a) GPS-only gravity field recovery with GOCE, CHAMP, and GRACE. Adv Space Res 47(6):1020-1028. doi:10.1016/j.asr.2010.11.008

Jäggi A, Prange L, Hugentobler U (2011b) Impact of covariance information of kinematic positions on orbit reconstruction and gravity field recovery. Adv Space Res 47(9):1472-1479. doi:10.1016/j.asr. 2010.12.009

Jakowski N, Mayer C, Wilken V (2006) GPS sounding of the ionosphere onboard CHAMP. In: Characterising the ionosphere, RTO-MP-IST056, Paper 26

Koop R, Gruber T, Rummel R (2006) The status of the GOCE high-level processing facility. In: 3rd GOCE User Workshop, 6-8 Nov 2006, Frascati, Italy, pp 195-205, ESA SP-627

Lemoine FG, Smith DE, Kunz L, Smith R, Pavlis EC, Pavlis NK, Klosko SM, Chinn DS, Torrence MH, Williamson RG, Cox CM, Rachlin KE, Wang YM, Kenyon SC, Salman R, Trimmer R, Rapp RH, Nerem RS (1997) The development of the NASA GSFC and NIMA joint geopotential model. In: Segawa J, Fujimoto H, Okubo S (eds) IAG symposia: gravity. Springer, Geoid and Marine Geodesy, pp 461-469

Lyard F, Lefevre F, Letellier T, Francis O (2006) Modelling the global ocean tides: insights from FES2004. Ocean Dynam 56:394-415. doi:10.1007/s10236-006-0086-x 
Mayer-Gürr T, Eicker A, Kurtenbach E, Ilk KH (2010) ITG-GRACE: Global static and temporal gravity field models from GRACE data. In Flechtner et al. (Eds) System Earth via GeodeticGeophysical Space Techniques. Springer, Berlin, pp 159-168. doi:10.1007/ 978-3-642-10228-8_13

Mayer-Gürr T, Rieser D, Hoeck E, Brockmann JM, Schuh W-D, Krasbutter I, Kusche J, Maier A, Krauss S, Hausleitner W, Baur O, Jäggi A, Meyer U, Prange L, Pail R, Fecher T, Gruber T (2012) The new combined satellite only model GOCO03s. In: International symposium on gravity, geoid and height systems GGHS 2012, Venice, Italy, October 09-12

Meyer U, Jäggi A, Beutler G (2012) Monthly gravity field solutions based on GRACE observations generated with the Celestial Mechanics Approach. Earth Planet Sci Lett 345-348:72-80. doi:10.1016/j. eps1.2012.06.026

McCarthy DD, Petit G (2004) IERS Conventions 2003. IERS Technical note no.32. Bundesamt für Kartographie und Geodäsie, Frankfurt am Main, Germany

Pail R, Bruinsma S, Migliaccio F, Förste C, Goiginger H, Schuh W-D, Höck E, Reguzzoni M, Brockmann JM, Abrikosov O, Veicherts M, Fecher T, Mayrhofer R, Krasbutter I, Sansò F, Tscherning CC (2011) First GOCE gravity field models derived by three different approaches. J Geod 85(11):819-843. doi:10.1007/ s00190-011-0467-x

Petit G, Luzum B (2010) IERS Conventions 2010. IERS Technical note no.36. Bundesamt für Kartographie und Geodäsie, Frankfurt am Main, Germany

Prange L (2010) Global gravity field determination using the GPS measurements made onboard the low earth orbiting satellite CHAMP. Geodätisch-geophysikalische Arbeiten in der Schweiz, vol 81. Schweizerische Geodätische Kommission
Reigber C, Lühr H, Schwintzer P (1998) Status of the CHAMP Mission. In: Rummel R, Drewes H, Bosch W, Hornik H (eds) Towards an integrated global geodetic observing system (IGGOS). Springer, Berlin, pp 63-65

Rummel R, Yi W, Stummer C (2011) GOCE gravitational gradiometry. J Geod 85(11):777-790. doi:10.1007/s00190-011-0500-0

Schaer S (1999) Mapping and predicting the earth's ionosphere using the global positioning system. Geodätisch-geophysikalische Arbeiten in der Schweiz, vol 59. Schweizerische Geodätische Kommission

Standish EM (1998) JPL Planetary and Lunar Ephemerides, DE405/LE405. JPL IOM 312.F-98-048

Švehla D, Rothacher M (2005) Kinematic precise orbit determination for gravity field determination. In: Sansò F (ed) A window on the future of geodesy. Springer, Berlin, pp 181-188. doi:10.1007/ 3-540-27432-4_32

Tapley BD, Bettadpur S, Ries JC, Thompson PF, Watkins M (2004) GRACE measurements of mass variability in the Earth system. Science 305(5683):503-505. doi:10.1126/science.1099192

van Gelderen M, Koop R (1997) The use of degree variances in satellite gradiometry. J Geod 71(6):337-343. doi:10.1007/s001900050101

van Helleputte T, Visser P (2008) GPS based orbit determination using accelerometer data. Aerosp Sci Technol 12(6):478-484. doi:10. 1016/j.ast.2007.11.002

Visser PNAM, van der Wal W, Schrama EJO, van den IJssel J, Bouman J, (2014) Assessment of observing time-variable gravity from GOCE GPS and accelerometer observations. J Geod. doi:10. 1007/s00190-014-0741-9

Weigelt M, van Dam T, Jäggi A, Prange L, Tourian MJ, Keller W, Sneeuw N (2013) Time-variable gravity signal in Greenland revealed by high-low satellite-to-satellite tracking. J Geophys Res Solid Earth 118(7):3848-3859. doi:10.1002/jgrb.50283 